\title{
Prognostic Value of Textural Features Obtained from F-fluorodeoxyglucose (F-18 FDG) Positron Emission Tomography/Computed Tomography (PET/CT) in Patients with Locally Advanced Cervical Cancer Undergoing Concurrent Chemoradiotherapy
}

Hyun-Woong Cho

Korea University Guro Hospital

\section{Eun Seong Lee}

Korea University Guro Hospital

Jae Kwan Lee

Korea University Guro Hospital

Jae Seon Eo

Korea University Guro Hospital https://orcid.org/0000-0003-3273-5661

Sungeun Kim

Korea University Anam Hospital

Jin Hwa Hong ( $\sim$ jhhong93@korea.ac.kr)

Korea University Guro Hospital https://orcid.org/0000-0002-6905-5363

\section{Research Article}

Keywords: Textural features, Prediction, Cervical cancer, Chemoradiotherapy

Posted Date: February 18th, 2022

DOI: https://doi.org/10.21203/rs.3.rs-1116901/v1

License: (c) (i) This work is licensed under a Creative Commons Attribution 4.0 International License.

Read Full License 


\section{Abstract \\ Background}

To evaluate whether textural features obtained from F-18 FDG PET/CT offer clinical value that can predict the outcome of patients with locally advanced cervical cancer (LACC) receiving concurrent chemoradiotherapy (CCRT).

\section{Methods}

We reviewed the records of 68 patients with stage IIB-IVA LACC who underwent PET/CT before CCRT. Conventional metabolic parameters, shape indices, and textural features of the primary tumor were measured on PET/CT. A Cox regression model was used to examine the effects of variables on overall survival (OS) and progression-free survival (PFS).

\section{Results}

PET/CT parameters were grouped into tertiles (low, middle, and high). In the high group, metabolic tumor volume [hazard ratio (HR), 18.94; 95\% confidence interval (Cl), 2.27-145.01], shape volume (HR, 21.77; $95 \% \mathrm{Cl}, 2.85-166.17)$, shape compacity ( $\mathrm{HR}, 10.53 ; 95 \% \mathrm{Cl}, 2.38-46.54)$, gray-level non-uniformity (GLNU) from grey-level run length matrix (GLRLM) (HR, 23.80; 95\% Cl, 3.13-181.31), and run-length nonuniformity from GLRLM (HR, 10.07; 95\% Cl, 2.28-44.48) were significant factors for poorer PFS. High coarseness from neighborhood grey-level different matrix (NGLDM) was associated with reduction of recurrence (HR, 0.09; 95\% Cl, 0.02-0.42).

\section{Conclusion}

Our results demonstrated that several features including textural indices on pretreatment PET/CT were strong prognostic factors for recurrence in patients with $L A C C$ receiving CCRT.

\section{Background}

Cervical cancer is one of most common cancers among women, with over 500,000 new cases annually worldwide resulting in over 270,000 deaths [1]. A significant proportion of patients are diagnosed at a locally advanced stage [2]. Concurrent chemo-radiotherapy (CCRT) became standard care for women with locally advanced cervical cancer (LACC) following a National Cancer Institute (NCl) alert based on the results of 5 randomized trials in 1999 [3-8]. However, approximately $30-40 \%$ of women with LACC are unable to achieve complete response after CCRT [9]. The International Federation of Gynecology and Obstetrics (FIGO) staging, histology, tumor size and lymph node (LN) metastases are known as 
prognostic factors, but even patients with the same clinical factors may have different clinical outcomes [10]. Therefore, novel markers to better identify patients at high risk of relapse are needed.

F-18 fluorodeoxyglucose (FDG) positron emission tomography/computed tomography (PET/CT) is essential in the initial evaluation of disease extent and in assessment of responses to CCRT in LACC.[11] Many studies have suggested that additional quantitative features available from PET/CT, such as standardized uptake value (SUV), metabolic tumor volume (MTV), and total lesion glycolysis (TLG), could provide predictive or prognostic information [12-19]. A recent meta-analysis of 12 studies involving cervical cancer demonstrated that the prognostic values of MTV and TLG for recurrence were consistently significant, with pooled hazard ratios (HRs) of 5.08-7.30 and 4.80-15.83, respectively [20]. However, these parameters do not reflect intratumor heterogeneity (ITH), which is one of the main causes of resistance to treatment [21, 22]. One of the most highlighted methods to quantify ITH is texture analysis from images [23]. Imaging analysis, including texture analysis, can be used to quantify tumor characteristics such as intensity, shape, and heterogeneity, some of which can provide clinically relevant and complementary information beyond conventional clinical characteristics in several cancers, including cervical cancer [24-29]. A number of recent studies have attempted textural analysis from PET/CT imaging to evaluate prognostic and predictive values after CCRT [10, 30, 31], but few studies have conducted a comprehensive analysis of clinical factors, conventional metabolic parameters, and textural features extracted from pretreatment PET/CT in LACC patients. Therefore, the purpose of this study was to evaluate whether the textural features obtained from F-18 FDG PET/CT imaging, based on comprehensive analysis, have clinical value that can predict the outcome of patients with LACC receiving CCRT.

\section{Methods}

\section{Patients}

Patients with histologically proven LACC, staged IIB-IVA (FIGO 2009 definition) and treated with definitive curative CCRT and subsequent brachytherapy from January 2011 to November 2018 (to ensure a minimum follow-up of 1 year) at two institutions were included in this retrospective study. Enrollment required: (a) histologically confirmed stage IIB-IVA LACC determined by the 2018 FIGO classifications, and (b) availability of follow-up information up to 24 months. Exclusion criteria were history of previous chemotherapy or radiotherapy and/or distant metastatic disease.

Pretreatment evaluation consisted of patient history evaluation and physical examination, gynecological evaluation with biopsy, complete blood analysis, urine analysis, chest radiography, sigmoidoscopy, pelvic magnetic resonance imaging (MRI), abdominopelvic CT, and F-18 FDG PET/CT. Cystoscopy was performed if necessary.

Medical records of patients including age and date of diagnosis, histology, 2018 FIGO stage, presence of positive LN on PET/CT, tumor size as measured on MRI, external beam radiotherapy (EBRT) and 
brachytherapy doses, and date and status (i.e., alive, deceased, and recurrence) at the last follow-up were retrieved. The date and site of recurrence were also collected.

\section{Treatment}

In patients scheduled for CCRT, a total of 54 Gy of external radiation (daily $2 \mathrm{~Gy}$ per fraction) was delivered. The radiation field was extended in patients with FDG uptake in the para-aortic lymph nodes. High-dose-rate brachytherapy was performed using 3.5 Gy per fraction, given in 8 fractions. Concurrent platinum-based chemotherapy was also administered to patients, and the chemotherapy regimens were as follows: weekly cisplatin $(n=41 ; 60.3 \%)$, paclitaxel-carboplatin $(n=19 ; 27.9 \%)$, and 5-FU-cisplatin $(n=$ 8; $11.8 \%)$.

\section{Follow-up}

After completion of treatment, follow-up examinations consisting of cervical smear, pelvic MRI with or without abdominopelvic CT, and serum tumor marker analysis were performed every 3 months for 2 years and at 6-month intervals for 3 years thereafter. Recurrence was confirmed by imaging studies or biopsy.

\section{PET/CT acquisition and image analysis}

Pretreatment PET/CT images were acquired using combined PET/CT scanners [Gemini TF/16 channel PET/CT scanner (Philips Medical Systems, Cleveland, OH, USA)] for all patients at the two participating institutions following routine clinical PET/CT protocols. After fasting for at least 6 hours, patients were given an intravenous injection of F-18 FDG; then, we waited approximately 60 minutes before scanning. A 1-min emission scan per bed position of PET scans was acquired immediately after unenhanced CT scan acquisition. The PET images were reconstructed to a $144 \times 144$ matrix size with a $4 \mathrm{~mm}$ slice thickness by means of an ordered subset expectation maximization algorithm incorporating time-of-flight information (3 iterations, 33 subsets).

LIFEx v.5.10 (https://www.lifexsoft.org) was used to delineate the volume of interest (VOI) of primary tumors and for extraction of each SUV metric and volumetric and radiomic feature [32]. The VOI on PET images was manually drawn with an SUV cut-off of 2.5 by a nuclear medicine physician who was blinded to patient clinical information. The following input parameters was used for calculation of features using LIFEx: $4.0 \mathrm{~mm}, 4.0 \mathrm{~mm}, 4.0 \mathrm{~mm}$ for spatial resampling; the number of gray levels in 64 bins for intensity discretization; absolute scale bounds between a minimum of 0 and a maximum of 25 for intensity rescaling.

\section{PET/CT parameters}

Conventional metabolic parameters [maximum SUV (SUVmax), MTV, and TLG], shape indices (sphericity, compacity, volume), histogram features (skewness, kurtosis, entropy, energy), and the four groups of textural features [grey level co-occurrence matrix (GLCM), grey-level run length matrix (GLRLM), grey-level zone length matrix (GLZLM), and neighborhood grey-level different matrix (NGLDM)] retrieved from pretreatment F-18 FDG PET/CT images were used in this study. 
The SUVmax was defined as the maximum SUV within the tumor and the MTV was defined as the FDG avid tumor volume measured bi-dimensionally at the longest diameter [33]. The TLG was calculated by multiplying the MTV with its corresponding mean SUV [34].

Sphericity is how VOI. Sphericity is equal to 1 for a perfect sphere. Compacity reflects how compact the VOI. Volume is the VOI in $\mathrm{mL}$ and in voxels.

GLCM takes into account the arrangements of pairs of voxels to calculate textural indices. The GLCM is calculated from 13 different directions in 3D with a $\delta$-voxel distance $(\|d\|)$ relationship between neighboured voxels. The index value is the average of the index over the 13 directions in space $(X, Y, Z)$. Seven textural indices are computed from this matrix.

NGLDM corresponds to the difference of grey-levels between one voxel and its 26 neighbours in 3 dimensions (8 in 2D). Three texture indices can be computed from this matrix.

GLRLM gives the size of homogeneous runs for each grey level. This matrix is computed for the 13 different directions in 3D (4 in 2D) and for each of the 11 texture indices derived from this matrix, the 3D value is the average over the 13 directions in 3D (4 in 2D). The element ( $i, j)$ of GLRLM corresponds to the number of homogeneous runs of $j$ voxels with intensity $i$ in an image and is called GLRLM(i, $j)$ thereafter.

GLZLM provides information on the size of homogeneous zones for each grey-level in 3 dimensions (or 2D). It is also named Grey Level Size Zone Matrix (GLSZM). From this matrix, 11 texture indices are computed. Element $(i, j)$ of GLZLM corresponds to the number of homogeneous zones of $j$ voxels with the intensity $i$ in an image and is called GLZLM $(i, j)$ thereafter.

Skewness is the the asymmetry of the grey-level distribution in the histogram. Kurtosis reflects the shape of the grey-level distribution (peaked or flat) relative to a normal distribution. Entropy reflects the randomness of the distribution. Energy reflects the uniformity of the distribution.

\section{Statistical analysis}

Categorical variables are presented as numbers and percentages, and continuous variables are presented as "median (range)". We used the Mann-Whitney $U$ test for analysis of continuous variables, and Chisquared test or Fisher's exact test for analysis of categorical variables. To examine correlations between $\mathrm{PET} / \mathrm{CT}$ parameters and disease recurrence, receiver operating characteristic $(\mathrm{ROC})$ curves were constructed to evaluate the optimal predictive performance among the various textural indices, conventional PET/CT parameters, and clinical parameters, such as stage, node metastasis, and tumor size.

Overall PET/CT parameters were grouped into tertile ranges (low, middle, and high groups) according to each parameter. For the high and low groups, survival curves were estimated according to the KaplanMeier method. Survival estimates were compared between the groups using the log-rank test. Univariate cox proportional hazards regression models were used to identify significant prognostic factors for 
progression-free survival (PFS) and overall survival (OS) followed by false discovery rate adjustments. OS time was calculated from treatment initiation to all deaths (cancer-related or not). PFS time was calculated from treatment initiation and tumor progression, or death from any cause. Associations are shown as HRs with $95 \%$ confidence intervals (Cls), and values of $P<0.05$ were considered statistically significant. All analyses were performed using the SAS statistical package (SAS Institute, Cary, NC, USA).

\section{Results}

\section{Patient characteristics and treatment outcomes}

The clinical characteristics of study participants are listed in Table 1. The median age was 60 (27-75) years. The predominant FIGO stage was IIIC. After a median follow-up of 49 (12-139) months, disease recurrence occurred in 22 patients (32.3\%), and 5 patients (7.4\%) died. Of the 22 patients who underwent disease recurrence, 17 had a local recurrence and 5 had a distant recurrence. There were no differences in age, parity, histology, and chemotherapy regimen in patients with and without recurrence. However, IIIC and IVA staging were more common in patients with recurrence compared with those who did not experience recurrence $(P=0.01)$. 
Table 1

Clinical characteristics in patients with or without recurrence

\begin{tabular}{|c|c|c|c|c|}
\hline Variables & $\begin{array}{l}\text { All } \\
(n=68)(\%)\end{array}$ & $\begin{array}{l}\text { No recurrence } \\
(n=46)(\%)\end{array}$ & $\begin{array}{l}\text { Recurrence } \\
(n=22)(\%)\end{array}$ & $p$ value \\
\hline Median age, year (range) & $60(27-75)$ & $59(34-78)$ & $59(27-75)$ & 0.38 \\
\hline Median parity (range) & $2(0-7)$ & $2(0-4)$ & $2(0-3)$ & 0.63 \\
\hline FIGO stage & & & & 0.01 \\
\hline IIB & $25(36.8)$ & $22(47.8)$ & $3(13.6)$ & \\
\hline IIIC & $31(45.6)$ & $19(41.3)$ & $12(54.5)$ & \\
\hline IVA & $12(17.6)$ & $5(10.9)$ & $7(31.8)$ & \\
\hline Histology & & & & 0.55 \\
\hline Squamous cell carcinoma & $61(89.7)$ & $41(89.1)$ & $20(90.9)$ & \\
\hline Adenocarcinoma & $4(5.9)$ & $2(4.3)$ & $2(9.1)$ & \\
\hline Mucinous carcinoma & $2(3.9)$ & $2(4.4)$ & 0 & \\
\hline Neuroendocrine carcinoma & $1(1.5)$ & $1(2.2)$ & & \\
\hline Radiotherapy & & & & 1.00 \\
\hline Whole pelvis (Gy) & & 54 & 54 & \\
\hline Brachytherapy (Gy) & & 28 & 28 & \\
\hline Chemotherapy & & & & 0.83 \\
\hline Weekly Cisplatin & $41(60.3)$ & $28(60.9)$ & $13(59.1)$ & \\
\hline Paclitaxel-Carboplatin & $19(27.9)$ & $12(26.1)$ & $7(31.8)$ & \\
\hline Cisplatin-5-fluorouracil & $8(11.8)$ & $6(13.0)$ & $2(9.1)$ & \\
\hline Recurrence & $22(32.3)$ & 0 & $22(100.0)$ & \\
\hline Death & $5(7.4)$ & 0 & $5(22.7)$ & \\
\hline
\end{tabular}

\section{Representative cases}

Two representative cases of the same cervical cancer stage with similar TLG have been shown in Figure 1. Figure 1 (A) shows F-18 FDG PET/CT images of cervical cancer patients with relatively high SUVmax, and relatively low textural values. On the other hand, Figure 1 (B) presents F-18 FDG PET/CT images of LACC patients with relatively low SUVmax and relatively high textural values. Although the stages were the same, the prognosis differed according to the ITH of the primary mass lesion. 


\section{Prognostic value of conventional metabolic parameters, shape descriptors, and textural features for PFS and OS}

The patients included in this study were classified into three groups based on tertiles of PET/CT parameters such as SUVmax, MTV, TLG, shape descriptors, and textural indices. Tertiles (as opposed to quartiles and quintiles.) were chosen to balance the flexibility gained by adding more groups with the need to keep group sizes sufficiently large for subgroup analyses.

As summarized in Table 2, Cox regression analysis indicated that the highest tertile group of several quantitative features from PET/CT is a significant prognostic factor compared with the lowest tertile group as a reference. Among conventional metabolic parameters, high MTV (HR, 18.94; 95\% Cl, 2.27145.01) was significant prognostic factors for inferior PFS after controlled by false discovery rate. The median PFS of patients with high and low MTV was 11.1 and 67.0 months, respectively $(P<0.001)$ (Figure 2). 
Table 2

Univariate cox regression model of clinical and PET/CT parameters controlled by false discovery rate for PFS and OS

\begin{tabular}{|c|c|c|c|c|c|c|}
\hline & PFS & & & 0 & & \\
\hline Variables & $\mathrm{HR}$ & $95 \% \mathrm{Cl}$ & $P$ value* & $\mathrm{HR}$ & $95 \% \mathrm{Cl}$ & $p$ value* \\
\hline Age $(>60)$ & 1.06 & $0.46-2.46$ & 0.7123 & 1.617 & $0.433-6.032$ & 0.4745 \\
\hline Parity & 0.892 & $0.64-1.25$ & 0.9776 & 1.089 & $0.688-1.723$ & 0.7168 \\
\hline Stage (III-IVA) & 4.457 & $1.311-15.150$ & 0.0823 & 2.524 & $0.523-12.175$ & 0.2487 \\
\hline Histology (SCC) & 0.765 & $0.178-3.286$ & 0.8804 & 0.889 & $0.111-7.112$ & 0.9114 \\
\hline Initial SCC-Ag (5ng/ml) & 2.543 & $0.854-7.566$ & 0.2261 & 2.167 & $0.45-10.448$ & 0.3351 \\
\hline SUVmean & 0.928 & $0.298-2.887$ & 0.6104 & 0.765 & $0.154-3.795$ & 0.9378 \\
\hline SUVstd & 0.951 & $0.333-2.720$ & 0.9776 & 0.759 & $0.153-3.766$ & 0.9246 \\
\hline SUVmax & 1.258 & $0.452-3.498$ & 0.9776 & 1.198 & $0.268-5.356$ & 0.6624 \\
\hline MTV & 18.94 & $2.27-145.01$ & $0.027^{\star}$ & 6.122 & $0.715-52.44$ & 0.1449 \\
\hline TLG & 9.055 & $2.033-40.321$ & 0.0521 & 5.885 & $0.687-50.413$ & 0.2347 \\
\hline HISTO_Skewness & 1.21 & $0.415-3.526$ & 0.9776 & 6.516 & $0.781-54.379$ & 0.1333 \\
\hline HISTO_Kurtosis & 1.365 & $0.455-4.095$ & 0.8559 & 5.403 & $0.628-46.513$ & 0.2796 \\
\hline HISTO_Entropy & 0.96 & $0.309-2.985$ & 0.8559 & 1.177 & $0.197-7.043$ & 0.9014 \\
\hline HISTO_Energy & 1.042 & $0.335-3.239$ & 0.5716 & 0.85 & $0.142-5.088$ & 0.9014 \\
\hline SHAPE_Volume & 21.769 & $2.852-166.173$ & $0.027 *$ & 7.767 & $0.933-64.684$ & 0.0978 \\
\hline SHAPE_Sphericity & 0.258 & $0.07-0.96$ & 0.2261 & 0.128 & $0.015-1.106$ & 0.1466 \\
\hline SHAPE_Compacity & 10.53 & $2.38-46.54$ & $0.027^{\star}$ & 7.072 & $0.85-58.805$ & 0.0973 \\
\hline GLCM_Homogeneity & 3.229 & $1.008-10.348$ & 0.3044 & 3.148 & $0.61-16.252$ & 0.2402 \\
\hline GLCM_Energy & 1.042 & $0.335-3.239$ & 0.5716 & 0.81 & $0.135-4.85$ & 0.8583 \\
\hline GLCM_Contrast & 0.503 & $0.166-1.524$ & 0.6719 & 0.654 & $0.146-2.925$ & 0.5903 \\
\hline GLCM_Correlation & 8.35 & $1.863-37.429$ & 0.0823 & 6.113 & $0.713-52.379$ & 0.2161 \\
\hline GLCM_Entropy & 1.135 & $0.38-3.386$ & 0.8383 & 1.215 & $0.203-7.273$ & 0.9203 \\
\hline GLCM_Dissimilarity & 0.405 & $0.138-1.19$ & 0.4331 & 0.489 & $0.117-2.048$ & 0.1855 \\
\hline GLRLM_SRE & 0.31 & $0.097-0.993$ & 0.3044 & 0.318 & $0.062-1.64$ & 0.2402 \\
\hline
\end{tabular}

* Statistical significance was considered at $p<0.05$ and was controlled by false discovery rate 


\begin{tabular}{|c|c|c|c|c|c|c|}
\hline & PFS & & & 0 & & \\
\hline GLRLM_LRE & 4.994 & $1.385-18.003$ & 0.1631 & 3.076 & $0.595-15.894$ & 0.2611 \\
\hline GLRLM_LGRE & 1.033 & $0.332-3.212$ & 0.5716 & 1.17 & $0.236-5.8$ & 0.9797 \\
\hline GLRLM_HGRE & 1.201 & $0.366-3.943$ & 0.3489 & 1.158 & $0.193-6.939$ & 0.8381 \\
\hline GLRLM_SRLGE & 0.568 & $0.185-1.739$ & 0.6657 & 0.764 & $0.128-4.573$ & 0.7946 \\
\hline GLRLM_SRHGE & 0.795 & $0.242-2.611$ & 0.332 & 1.177 & $0.197-7.043$ & 0.9014 \\
\hline GLRLM_LRLGE & 1.552 & $0.491-4.906$ & 0.5716 & 1.95 & $0.326-11.675$ & 0.7382 \\
\hline GLRLM_LRHGE & 1.635 & $0.535-5.000$ & 0.8209 & 0.851 & $0.172-4.222$ & 0.9534 \\
\hline GLRLM_GLNU & 23.80 & $3.13-181.31$ & $0.027 *$ & 8.907 & $1.094-72.483$ & 0.0781 \\
\hline GLRLM_RLNU & 10.071 & $2.28-44.48$ & $0.027 *$ & 5.926 & $0.692-50.763$ & 0.2337 \\
\hline GLRLM_RP & 0.231 & $0.063-0.842$ & 0.2178 & 0.331 & $0.064-1.707$ & 0.2756 \\
\hline NGLDM_Coarseness & 0.09 & $0.02-0.42$ & $0.027 *$ & 0.141 & $0.017-1.169$ & 0.1122 \\
\hline NGLDM_Contrast & 0.266 & $0.084-0.843$ & 0.1883 & 0.125 & $0.015-1.035$ & 0.0624 \\
\hline NGLDM_Busyness & 7.683 & $1.706-34.597$ & 0.1203 & 2.178 & $0.396-11.981$ & 0.6681 \\
\hline GLZLM_SZE & 0.915 & $0.329-2.545$ & 0.9776 & 0.746 & $0.166-3.34$ & 0.7597 \\
\hline GLZLM_LZE & 7.301 & $1.604-33.221$ & 0.1495 & 3.105 & $0.601-16.038$ & 0.261 \\
\hline GLZLM_LGZE & 1.09 & $0.392-3.03$ & 0.9776 & 1.2 & $0.242-5.951$ & 0.9752 \\
\hline GLZLM_HGZE & 1.15 & $0.400-3.307$ & 0.9321 & 1.747 & $0.32-9.546$ & 0.8015 \\
\hline GLZLM_SZLGE & 1.18 & $0.425-3.296$ & 0.9776 & 0.877 & $0.146-5.257$ & 0.7885 \\
\hline GLZLM_SZHGE & 1.158 & $0.403-3.328$ & 0.9321 & 1.284 & $0.287-5.75$ & 0.6827 \\
\hline GLZLM_LZLGE & 5.085 & $1.411-18.325$ & 0.1602 & 3.831 & $0.772-19.01$ & 0.1236 \\
\hline GLZLM_LZHGE & 6.288 & $1.363-29.005$ & 0.1883 & 4.52 & $0.504-40.577$ & 0.402 \\
\hline GLZLM_GLNU & 5.823 & $1.655-20.492$ & 0.0521 & 2.586 & $0.501-13.341$ & 0.31 \\
\hline GLZLM_ZLNU & 2.948 & $1.005-8.645$ & 0.1948 & 1.544 & $0.343-6.944$ & 0.424 \\
\hline GLZLM_ZP & 0.266 & $0.072-0.985$ & 0.2241 & 0.313 & $0.061-1.616$ & 0.2281 \\
\hline
\end{tabular}

For shape descriptors, high volume (HR, 21.77; $95 \% \mathrm{Cl}, 2.85-166.17)$ and compacity $(\mathrm{HR}, 10.53 ; 95 \% \mathrm{Cl}$, 2.38-46.54) revealed high prognostic value with statistical significance. The median PFS of patients with low volume (high vs low: 16.8 vs 48.7 months, $\mathrm{P}<0.001$ ) and compacity (high vs low: 11.1 vs 48.7 
months, $\mathrm{P}<0.001$ ) was significantly higher than those of patients with high volume and compacity (Figure 3).

Among the textural features, three were significant prognostic factors for lowering PFS when controlled by false discovery rate (Table 2); they are listed as follows: high GLNU (HR, 23.80; 95\% Cl, 3.13-181.31), and RLNU (HR, 10.07; 95\% Cl, 2.28-44.48) from GLRLM; low coarseness from NGLDM (HR, 0.09; 95\% Cl, $0.02-0.42$ ). The median PFS are as follows (Figure 4): high vs. low GLNU derived from GLRLM, 8.6 vs. 57.7 months $(P<0.001)$; high vs. low RLNU derived from GLRLM, 16.8 vs. 48.7 months $(P=0.001)$; high vs. low coarseness derived from NGLDM, 48.7 vs. 11.1 months $(P<0.001)$. No clinical factors or features with significant prognostic value for patient OS were extracted from the PET/CT images.

\section{Predictive ability of different clinical factors, metabolic parameters, shape descriptors, and textural indices for local failures and distant metastasis}

The area under the ROC curve was reported for the parameters extracted from PET/CT images, which were identified as significant prognostic factors for PFS, using Cox regression analysis (Table 3). Five PET/CT parameters, namely, shape descriptors (volume and compacity), MTV, GLNU from GLRLM, and coarseness from NGLDM, were good predictors of post-CCRT recurrence, with an area under curve (AUC) $>0.75$. Among conventional metabolic parameters, MTV (AUC 0.7808, P $<0.001)$ had the most accurate predictive value for recurrence. Among shape descriptors, compacity (AUC 0.804, P < 0.001) was the best predictor. Among textural indices, GLNU derived from GLRLM most accurately and consistently predicted tumor relapse (AUC 0.7808, $\mathrm{P}<0.001$ ). In terms of clinical factors, advanced stage (III-IVA) was the most effective predictor for recurrence (AUC 0.714, $\mathrm{P}<0.001$ ) 
Table 3

Predictive textural indices and clinical factors for recurrence and the area under the ROC Curve

\begin{tabular}{|llll|}
\hline Variables & AUC & $95 \% \mathrm{Cl}$ & $\boldsymbol{p}$ value \\
\hline SHAPE_Compacity & 0.804 & $0.7031-0.905$ & $<0.001$ \\
\hline SHAPE_Volume & 0.7848 & $0.6813-0.8884$ & $<0.001$ \\
\hline MTV & 0.7808 & $0.6761-0.8855$ & $<0.001$ \\
\hline GLRLM_GLNU & 0.7808 & $0.6761-0.8855$ & $<0.001$ \\
\hline NGLDM_Coarseness & 0.75 & $0.6349-0.8651$ & $<0.001$ \\
\hline TLG & 0.7354 & $0.6182-0.8525$ & $<0.001$ \\
\hline GLRLM_RLNU & 0.7354 & $0.6182-0.8525$ & $<0.001$ \\
\hline Stage (III-IVA) & 0.714 & $0.592-0.817$ & $<0.001$ \\
\hline GLCM_Correlation & 0.7121 & $0.5929-0.8314$ & $<0.001$ \\
\hline NGLDM_Busyness & 0.7121 & $0.5929-0.8314$ & $<0.001$ \\
\hline GLZLM_GLNU & 0.6899 & $0.5634-0.8164$ & 0.002 \\
\hline Nodal status & 0.67 & $0.545-0.779$ & 0.002 \\
\hline GLZLM_LZHGE & 0.6667 & $0.5392-0.7942$ & 0.003 \\
\hline GLZLM_ZLNU & 0.6556 & $0.5185-0.7927$ & 0.003 \\
\hline GLZLM_LZE & 0.65 & $0.5177-0.7823$ & 0.003 \\
\hline Tumor size ( $\geq 2 c m)$ & 0.649 & $0.524-0.761$ & 0.004 \\
\hline SHAPE_Sphericity & 0.6495 & $0.5219-0.7771$ & 0.002 \\
\hline GLRLM_LRE & 0.6495 & $0.5219-0.7771$ & 0.004 \\
\hline GLRLM_RP & 0.6424 & $0.511-0.7739$ & 0.004 \\
\hline
\end{tabular}

\section{Discussion}

The present study demonstrated that six features derived from PET/CT images are powerful predictors for recurrence in LACC patients receiving CCRT: MTV, shape descriptor volume and compacity; GLNU, and RLNU from GLRLM; and coarseness from NGLDM. Our finding that several textural features have predictive value for prognosis in LACC patients suggests that ITH is crucial in responses to cancer treatment. Based on such results, there might be a need for a validated prediction model incorporating textural features from PET/CT images. 
In this study, we performed comprehensive analysis involving 40 features, including textural indices extracted from pretreatment PET/CT images, to identify independent prognostic markers in patients with LACC. Recently, few previous studies conducted comprehensive analysis incorporating textural features from PET/CT images related to prognosis in LACC patients who received CCRT $[2,10,35,36]$. Lucia et al. demonstrated that GLNU from GLRLM in PET/CT and entropy from GLCM in MRI were identified as independent prognostic factors [2]. Subsequently, the authors developed a PET/MRI predictive model with textural features and validated it successfully in independent external cohorts [10]. Recently, Mu et al. showed the radiomics nomograms constructed with $T$ stage, lymph node status, and radiomics signatures that can predict OS and PFS better than FIGO stage [35]. Esfahani et al. demonstrated that significant difference was seen between eight features in local and metastatic tumors including MTV, TLG, and entropy on PET from PET/CT [36]. All previous studies, including this, were small, and textural analysis with PET/CT can be influenced by factors that require interinstitutional standardization, such as image acquisition, reconstruction, preprocessing, segmentation, and mathematical methods [37]. Therefore, further studies are necessary.

Our findings suggest that certain shape descriptors or textural features may predict recurrence of LACC better than conventional SUV parameters. Three textural features and two shape descriptors were significant risk factors for recurrence of LACC after CCRT, whereas only MTV from conventional metabolic parameters exhibited significant prognostic value in this study. In addition, the two shape descriptors had the highest predictive value for recurrence in ROC analysis, and GLNU from GLRLM had a similar predictive value compared to MTV. The results of this study have clinical implications for personalized treatment in patients who do not respond well to CCRT during the course of early treatment.

These results are in accord with those of other studies surrounding cervical cancer and relying on PET/CT. In a retrospective cohort study of 142 patients with LACC, high gray-level run emphasis (HGRE) derived from GLRLM could predict the presence of pelvic residual or recurrent tumors most accurately, and a low HGRE (HR, 4.34; $\mathrm{P}<0.0001)$ were the only prognostic factors among PET/CT parameters for low OS [31]. In another cohort study, only GLNU from GLRLM was an independent predictor of recurrence and loco-regional control with significantly higher prognostic power than conventional metabolic parameters such as SUVmax and clinical factors [2]. On the other hand, Chong et al. suggested that heterogeneity factors (AUC, $0.632 ; 95 \% \mathrm{Cl}, 0.526-0.739$ ) measured from PET/CT images do not offer superiority over conventional metabolic parameters (SUVmax: AUC, $0.732 ; 95 \% \mathrm{Cl}, 0.632-0.833$ ) for predicting disease recurrence [30].

Our results suggest that shape descriptor volume and compacity are strong prognostic factors for predicting cancer recurrence. Lucia et al. extracted 19 shape-based features to evaluate their predictive values for recurrence or loco-regional control, but only volume factor showed borderline significance (HR, 2.09; $95 \% \mathrm{Cl}, 0.94-4.62 ; \mathrm{P}=0.06$ ) [2]. Another cohort revealed that distant metastasis was significantly associated with greater average tumor volume [38]. Although no study has reported a relationship between compacity and prognosis in cervical cancer, few studies have shown that compacity is associated with several tumor types, including pelvic malignancies [39-41]. 
This study also demonstrated that MTV are powerful predictors of recurrence. Many studies have shown an association between conventional metabolic parameters and prognosis in cervical cancer. In a Korean study of cervical cancer patients with stage IB to IIA disease, tumor recurrence was significantly associated with TLG $(P<0.001)$, MTV $(P=0.001)$, and SUV $(P=0.004)$.[24] A retrospective study demonstrated that SUVtotal and MTVtotal were independent prognostic factors for OS in 510 cervical cancer patients staged IB to IVB [42]. Another prospective study suggested that a decline in TLG (<60\%) was a significant independent predictor of OS in patients with bulky $(\geq 4 \mathrm{~cm})(P=0.009)$ cervical cancer [26]. In addition, metabolic burdens (MTV and TLG) were beneficial for predicting prognosis in patients with cervical squamous cell carcinoma $[43,44]$.

This study demonstrated comprehensive textural analysis with four groups of textural indices extracted from PET/CT images that had strong predictive values for recurrence, even after controlling for clinical factors such as tumor stage and size. Nevertheless, our study had certain limitations. The findings of the present study should be interpreted cautiously because they represent a retrospective study design. This study involved a relatively small cohort size and a limited number of recurrences or deaths. In addition, validation was not performed in this study. Thus, external validation with a larger independent cohort will be necessary. Finally, because textural indices from PET/CT imaging are dependent on various factors such as image acquisition, reconstruction, preprocessing, segmentation, and mathematical methods, standardization of textural analysis will be necessary in the future [37].

\section{Conclusion}

Our results demonstrated that several features, including textural indices on pretreatment F-18 FDG PET/CT images, were significant prognostic factors for recurrence in patients with LACC receiving CCRT. Based on our preliminary results, further independent validation studies should be conducted to identify high-risk patients at diagnosis using F-18 FDG PET/CT.

\section{Abbreviations}

F-18 FDG: F-fluorodeoxyglucose; PET/CT: positron emission tomography/computed tomography; LACC: locally advanced cervical cancer; CCRT: concurrent chemoradiotherapy; OS: overall survival (OS); PFS: progression-free survival; HR: hazard ratio; Cl: confidence interval; GLNU: gray-level non-uniformity; GLRLM: grey-level run length matrix; NGLDM: neighborhood grey-level different matrix; NCl: National Cancer Institute; FIGO: International Federation of Gynecology and Obstetrics; LN: lymph node; SUV: standardized uptake value; MTV: metabolic tumor volume; TLG: total lesion glycolysis; ITH: intratumor heterogeneity; MRI: magnetic resonance imaging: EBRT: external beam radiotherapy; VOI: volume of interest; GLCM: grey level co-occurrence matrix (GLCM); GLZLM: grey-level zone length matrix; ROC: receiver operating characteristic; AUC: area under curve.

\section{Declarations}


Ethics approval and consent to participate

This study was approved by the medical research review committee of Korea University Guro Hospital (IRB approval number: 2019GR0318).Informed consent was waived because of the retrospective nature of the study.

Consent for publication All the authors consent for publication if accepted.

\section{Availability of data and materials}

The datasets used and/or analyzed during the current study are available fromthe corresponding author on reasonable request.

\section{Competing interest}

The authors declare that they have no competing interests.

\section{Funding}

No funding

\section{Authors' contribution}

Protocol/project development: C.H.W., L.E.S., E.J.S., H.J.H.

Data collection or management: C.H.W., L.E.S., E.J.S., H.J.H., K.S.

Data analysis: C.H.W., L.E.S.

Manuscript writing/editing: C.H.W., L.E.S., E.J.S., H.J.H., K.S.

\section{Acknowledgements}

We are thankful to the data providers for sharing their precious resources.

\section{References}

1. Ferlay J, Soerjomataram I, Dikshit R, Eser S, Mathers C, Rebelo M, Parkin DM, Forman D, Bray F: Cancer incidence and mortality worldwide: sources, methods and major patterns in GLOBOCAN 2012. Int J Cancer 2015, 136(5):E359-E386.

2. Lucia F, Visvikis D, Desseroit MC, Miranda O, Malhaire JP, Robin P, Pradier O, Hatt M, Schick U: Prediction of outcome using pretreatment (18)F-FDG PET/CT and MRI radiomics in locally advanced cervical cancer treated with chemoradiotherapy. Eur J Nucl Med Mol Imaging 2018, 45(5):768-786. 
3. Whitney CW, Sause W, Bundy BN, Malfetano JH, Hannigan EV, Fowler WC, Jr., Clarke-Pearson DL, Liao SY: Randomized comparison of fluorouracil plus cisplatin versus hydroxyurea as an adjunct to radiation therapy in stage IIB-IVA carcinoma of the cervix with negative para-aortic lymph nodes: a Gynecologic Oncology Group and Southwest Oncology Group study. J Clin Oncol 1999, 17(5):1339-1348.

4. Morris M, Eifel PJ, Lu J, Grigsby PW, Levenback C, Stevens RE, Rotman M, Gershenson DM, Mutch DG: Pelvic radiation with concurrent chemotherapy compared with pelvic and para-aortic radiation for highrisk cervical cancer. N Engl J Med 1999, 340(15):1137-1143.

5. Rose PG, Bundy BN, Watkins EB, Thigpen JT, Deppe G, Maiman MA, Clarke-Pearson DL, Insalaco S: Concurrent cisplatin-based radiotherapy and chemotherapy for locally advanced cervical cancer. N Engl J Med 1999, 340(15):1144-1153.

6. Keys HM, Bundy BN, Stehman FB, Muderspach LI, Chafe WE, Suggs CL, 3rd, Walker JL, Gersell D: Cisplatin, radiation, and adjuvant hysterectomy compared with radiation and adjuvant hysterectomy for bulky stage IB cervical carcinoma. N Engl J Med 1999, 340(15):1154-1161.

7. Peters WA, 3rd, Liu PY, Barrett RJ, 2nd, Stock RJ, Monk BJ, Berek JS, Souhami L, Grigsby P, Gordon W, Jr., Alberts DS: Concurrent chemotherapy and pelvic radiation therapy compared with pelvic radiation therapy alone as adjuvant therapy after radical surgery in high-risk early-stage cancer of the cervix. $J$ Clin Oncol 2000, 18(8):1606-1613.

8. Thomas GM: Improved treatment for cervical cancer-concurrent chemotherapy and radiotherapy. $N$ Engl J Med 1999, 340(15):1198-1200.

9. Kumar L, Gupta S: Integrating Chemotherapy in the Management of Cervical Cancer: A Critical Appraisal. Oncology 2016, 91 Suppl 1:8-17.

10. Lucia F, Visvikis D, Vallières M, Desseroit MC, Miranda O, Robin P, Bonaffini PA, Alfieri J, Masson I, Mervoyer A et al: External validation of a combined PET and MRI radiomics model for prediction of recurrence in cervical cancer patients treated with chemoradiotherapy. Eur J Nucl Med Mol Imaging 2019, 46(4):864-877.

11. Herrera FG, Prior JO: The role of PET/CT in cervical cancer. Front Onco/ 2013, 3:34.

12. Boellaard R, O'Doherty MJ, Weber WA, Mottaghy FM, Lonsdale MN, Stroobants SG, Oyen WJ, Kotzerke J, Hoekstra OS, Pruim J et al: FDG PET and PET/CT: EANM procedure guidelines for tumour PET imaging: version 1.0. Eur J Nucl Med Mol Imaging 2010, 37(1):181-200.

13. Choi ES, Ha SG, Kim HS, Ha JH, Paeng JC, Han I: Total lesion glycolysis by 18F-FDG PET/CT is a reliable predictor of prognosis in soft-tissue sarcoma. Eur J Nucl Med Mol Imaging 2013, 40(12):18361842. 
14. Hyun SH, Ahn HK, Kim H, Ahn MJ, Park K, Ahn YC, Kim J, Shim YM, Choi JY: Volume-based assessment by (18)F-FDG PET/CT predicts survival in patients with stage III non-small-cell lung cancer. Eur J Nucl Med Mol Imaging 2014, 41(1):50-58.

15. Klabatsa A, Chicklore S, Barrington SF, Goh V, Lang-Lazdunski L, Cook GJ: The association of 18FFDG PET/CT parameters with survival in malignant pleural mesothelioma. Eur J Nucl Med Mol Imaging 2014, 41(2):276-282.

16. Maffione AM, Ferretti A, Grassetto G, Bellan E, Capirci C, Chondrogiannis S, Gava M, Marzola MC, Rampin L, Bondesan C et al: Fifteen different 18F-FDG PET/CT qualitative and quantitative parameters investigated as pathological response predictors of locally advanced rectal cancer treated by neoadjuvant chemoradiation therapy. Eur J Nucl Med Mol Imaging 2013, 40(6):853-864.

17. Ryu IS, Kim JS, Roh JL, Cho KJ, Choi SH, Nam SY, Kim SY: Prognostic significance of preoperative metabolic tumour volume and total lesion glycolysis measured by (18)F-FDG PET/CT in squamous cell carcinoma of the oral cavity. Eur J Nucl Med Mol Imaging 2014, 41(3):452-461.

18. Wahl RL, Jacene H, Kasamon Y, Lodge MA: From RECIST to PERCIST: Evolving Considerations for PET response criteria in solid tumors. J Nucl Med 2009, 50 Suppl 1(Suppl 1):122S-150S.

19. Young H, Baum R, Cremerius U, Herholz K, Hoekstra O, Lammertsma AA, Pruim J, Price P: Measurement of clinical and subclinical tumour response using [18F]-fluorodeoxyglucose and positron emission tomography: review and 1999 EORTC recommendations. European Organization for Research and Treatment of Cancer (EORTC) PET Study Group. Eur J Cancer 1999, 35(13):1773-1782.

20. Han S, Kim H, Kim YJ, Suh CH, Woo S: Prognostic Value of Volume-Based Metabolic Parameters of (18)F-FDG PET/CT in Uterine Cervical Cancer: A Systematic Review and Meta-Analysis. AJR Am J Roentgenol 2018, 211(5):1112-1121.

21. Gerlinger M, Rowan AJ, Horswell S, Math M, Larkin J, Endesfelder D, Gronroos E, Martinez P, Matthews N, Stewart A et al: Intratumor heterogeneity and branched evolution revealed by multiregion sequencing. N Engl J Med 2012, 366(10):883-892.

22. Gerlinger M, Swanton C: How Darwinian models inform therapeutic failure initiated by clonal heterogeneity in cancer medicine. Br J Cancer 2010, 103(8):1139-1143.

23. Ha S, Choi H, Paeng JC, Cheon GJ: Radiomics in Oncological PET/CT: a Methodological Overview. Nucl Med Mol Imaging 2019, 53(1):14-29.

24. Chung HH, Kang SY, Ha S, Kim JW, Park NH, Song YS, Cheon GJ: Prognostic value of preoperative intratumoral FDG uptake heterogeneity in early stage uterine cervical cancer. J Gynecol Oncol2016, 27(2):e15. 
25. Guan Y, Li W, Jiang Z, Chen Y, Liu S, He J, Zhou Z, Ge Y: Whole-Lesion Apparent Diffusion CoefficientBased Entropy-Related Parameters for Characterizing Cervical Cancers: Initial Findings. Acad Radio/2016, 23(12):1559-1567.

26. Ho KC, Fang YH, Chung HW, Yen TC, Ho TY, Chou HH, Hong JH, Huang YT, Wang CC, Lai CH: A preliminary investigation into textural features of intratumoral metabolic heterogeneity in (18)F-FDG PET for overall survival prognosis in patients with bulky cervical cancer treated with definitive concurrent chemoradiotherapy. Am J Nucl Med Mol Imaging 2016, 6(3):166-175.

27. Reuzé S, Orlhac F, Chargari C, Nioche C, Limkin E, Riet F, Escande A, Haie-Meder C, Dercle L, Gouy S et al: Prediction of cervical cancer recurrence using textural features extracted from 18F-FDG PET images acquired with different scanners. Oncotarget 2017, 8(26):43169-43179.

28. Torheim T, Groendahl AR, Andersen EK, Lyng H, Malinen E, Kvaal K, Futsaether CM: Cluster analysis of dynamic contrast enhanced MRI reveals tumor subregions related to locoregional relapse for cervical cancer patients. Acta Oncol 2016, 55(11):1294-1298.

29. Yip SS, Aerts HJ: Applications and limitations of radiomics. Phys Med Bio/ 2016, 61(13):R150-166.

30. Chong GO, Lee WK, Jeong SY, Park SH, Lee YH, Lee SW, Hong DG, Kim JC, Lee YS: Prognostic value of intratumoral metabolic heterogeneity on F-18 fluorodeoxyglucose positron emission tomography/computed tomography in locally advanced cervical cancer patients treated with concurrent chemoradiotherapy. Oncotarget 2017, 8(52):90402-90412.

31. Chen SW, Shen WC, Hsieh TC, Liang JA, Hung YC, Yeh LS, Chang WC, Lin WC, Yen KY, Kao CH: Textural features of cervical cancers on FDG-PET/CT associate with survival and local relapse in patients treated with definitive chemoradiotherapy. Sci Rep 2018, 8(1):11859.

32. Nioche C, Orlhac F, Boughdad S, Reuze S, Goya-Outi J, Robert C, Pellot-Barakat C, Soussan M, Frouin F, Buvat I: LIFEx: A Freeware for Radiomic Feature Calculation in Multimodality Imaging to Accelerate Advances in the Characterization of Tumor Heterogeneity. Cancer Res 2018, 78(16):4786-4789.

33. Herrera FG, Breuneval T, Prior JO, Bourhis J, Ozsahin M: [(18)F]FDG-PET/CT metabolic parameters as useful prognostic factors in cervical cancer patients treated with chemo-radiotherapy. Radiat Oncol 2016, 11:43.

34. McDonald JE, Kessler MM, Gardner MW, Buros AF, Ntambi JA, Waheed S, van Rhee F, Zangari M, Heuck CJ, Petty N et al: Assessment of Total Lesion Glycolysis by (18)F FDG PET/CT Significantly Improves Prognostic Value of GEP and ISS in Myeloma. Clin Cancer Res 2017, 23(8):1981-1987.

35. Mu W, Liang Y, Hall LO, Tan Y, Balagurunathan Y, Wenham R, Wu N, Tian J, Gillies RJ: (18)F-FDG PET/CT Habitat Radiomics Predicts Outcome of Patients with Cervical Cancer Treated with Chemoradiotherapy. Radiol Artif Intell 2020, 2(6):e190218. 
36. Esfahani SA, Torrado-Carvajal A, Amorim BJ, Groshar D, Domachevsky L, Bernstine H, Stein D, Gervais D, Catalano OA: PET/MRI and PET/CT Radiomics in Primary Cervical Cancer: A Pilot Study on the Correlation of Pelvic PET, MRI, and CT Derived Image Features. Mol Imaging Biol 2021.

37. Hatt M, Tixier F, Pierce L, Kinahan PE, Le Rest CC, Visvikis D: Characterization of PET/CT images using texture analysis: the past, the present... any future? Eur J Nucl Med Mol Imaging 2017, 44(1):151165.

38. Altazi BA, Fernandez DC, Zhang GG, Hawkins S, Naqvi SM, Kim Y, Hunt D, Latifi K, Biagioli M, Venkat $P$ et al: Investigating multi-radiomic models for enhancing prediction power of cervical cancer treatment outcomes. Phys Med 2018, 46:180-188.

39. Crandall J, Fraum T, Lee M-Y, Grigsby P, Wahl R: Repeatability of 18F-FDG PET radiomic features in pelvic malignancies. J Nucl Med 2020, 61 Suppl1(supplement 1):272.

40. Novikov M: Multiparametric quantitative and texture (18)F-FDG PET/CT analysis for primary malignant tumour grade differentiation. Eur Radiol Exp 2019, 3(1):48.

41. Sollini M, Cozzi L, Pepe G, Antunovic L, Lania A, Di Tommaso L, Magnoni P, Erba PA, Kirienko M: [(18)F]FDG-PET/CT texture analysis in thyroid incidentalomas: preliminary results. Eur $J$ Hybrid Imaging 2017, 1(1):3.

42. Cegla P, Burchardt E, Roszak A, Czepczynski R, Kubiak A, Cholewinski W: Influence of Biological Parameters Assessed in [18F]FDG PET/CT on Overall Survival in Cervical Cancer Patients. Clin Nucl Med 2019, 44(11):860-863.

43. Rahman T, Tsujikawa T, Yamamoto M, Chino Y, Shinagawa A, Kurokawa T, Tsuchida T, Kimura H, Yoshida Y, Okazawa H: Different Prognostic Implications of 18F-FDG PET Between Histological Subtypes in Patients With Cervical Cancer. Medicine (Baltimore) 2016, 95(9):e3017.

44. Tsujikawa T, Rahman T, Yamamoto M, Yamada S, Tsuyoshi H, Kiyono Y, Kimura H, Yoshida Y, Okazawa H: (18)F-FDG PET radiomics approaches: comparing and clustering features in cervical cancer. Ann Nucl Med 2017, 31(9):678-685.

\section{Figures}




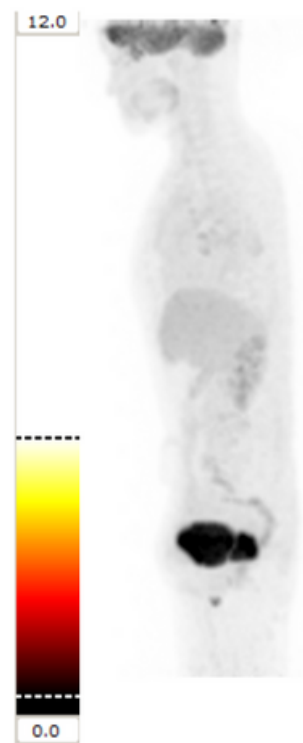

(a)

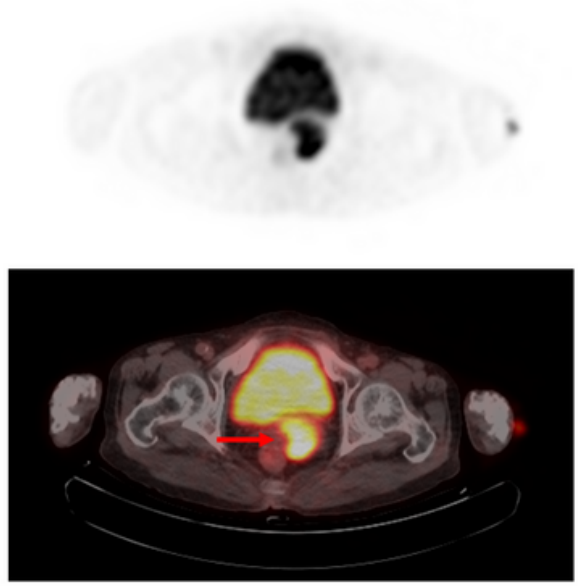

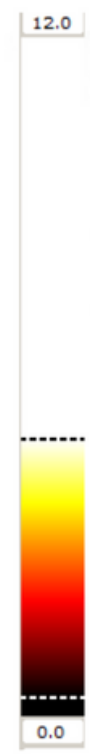

(b)

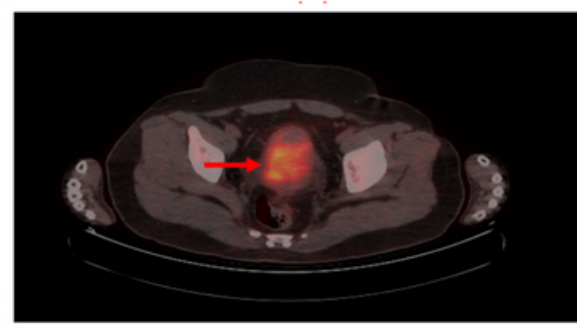

\section{Figure 1}

Representative cases of cervical cancer. (A) F-18 FDG PET/CT images of 47-year-old woman with stage IIB cervical squamous cell carcinoma. Trans-axial PET/CT and PET images show a mass lesion (red arrow) with high uptake value (SUVmax, 13.7;TLG, 321.1) and low heterogeneous distribution of FDG (shape descriptor compacity, 1.85; GLNU from GLRLM, 27.6). The patient had no recurrence for 3 years. (B)F-18 FDG PET/CT images of 42-year-old woman with stage IIB cervical squamous cell carcinoma. Trans-axial PET/CT and PET imagingreveal a mass lesion (red arrow) with relatively low uptake value (SUVmax, 8.4;TLG, 329.7) and a relatively high heterogeneous distribution of FDG (shape descriptor compacity, 2.33; GLNU from GLRLM,94.4). The patient recurred 7 months after CCRT and subsequently died. 


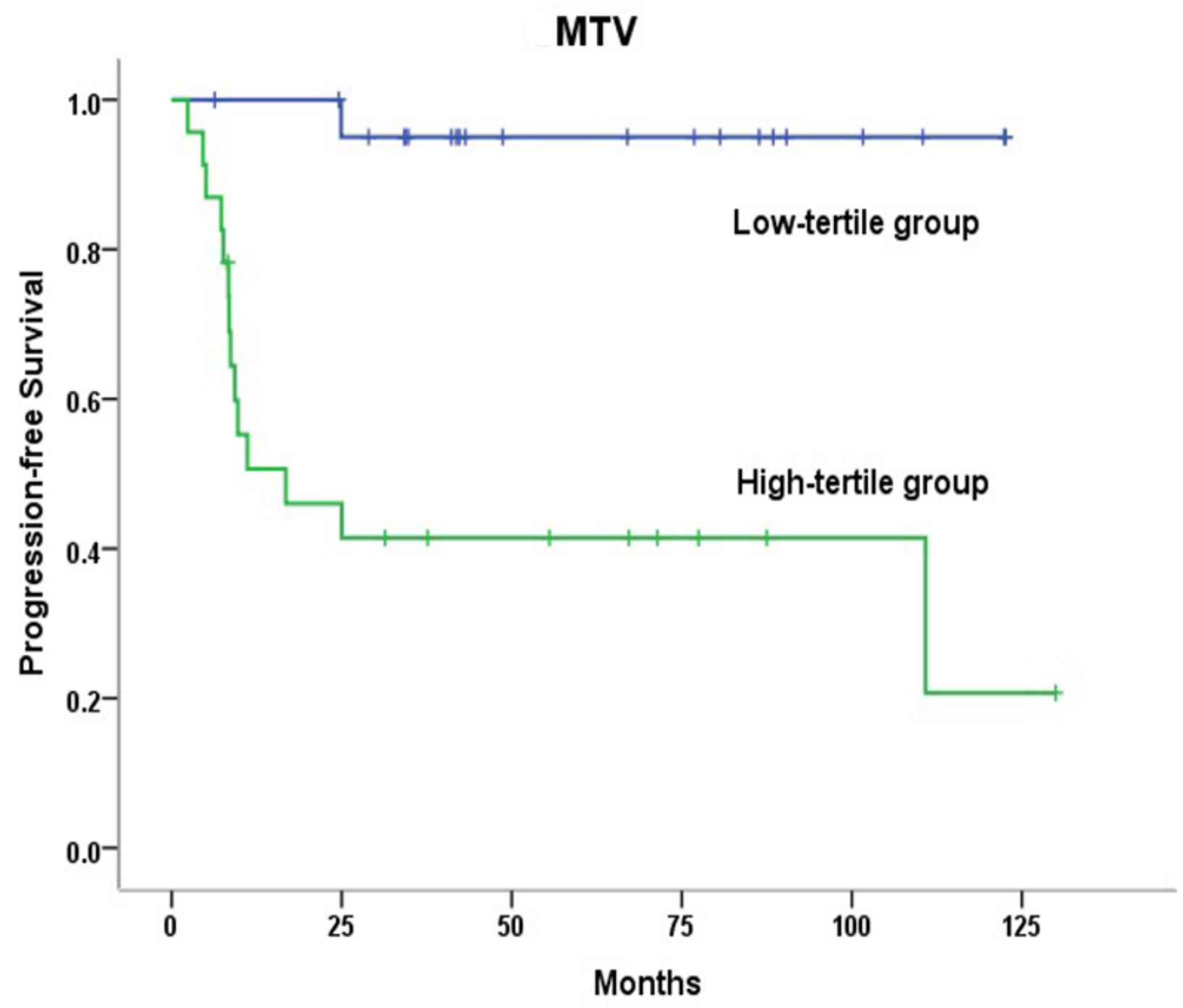

Figure 2

Progression-free survival of patients with MTV. 

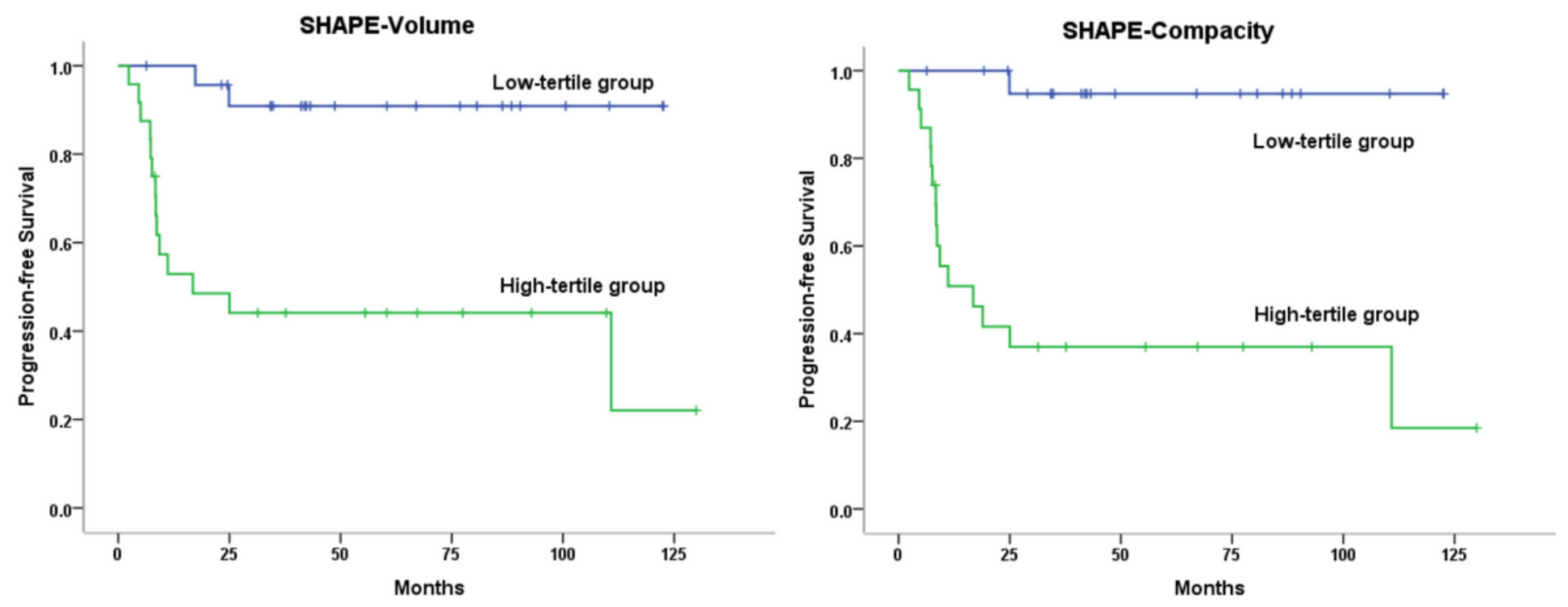

Figure 3

Progression-free survival of patients with low and high shape-volumeandshape-compacity. 

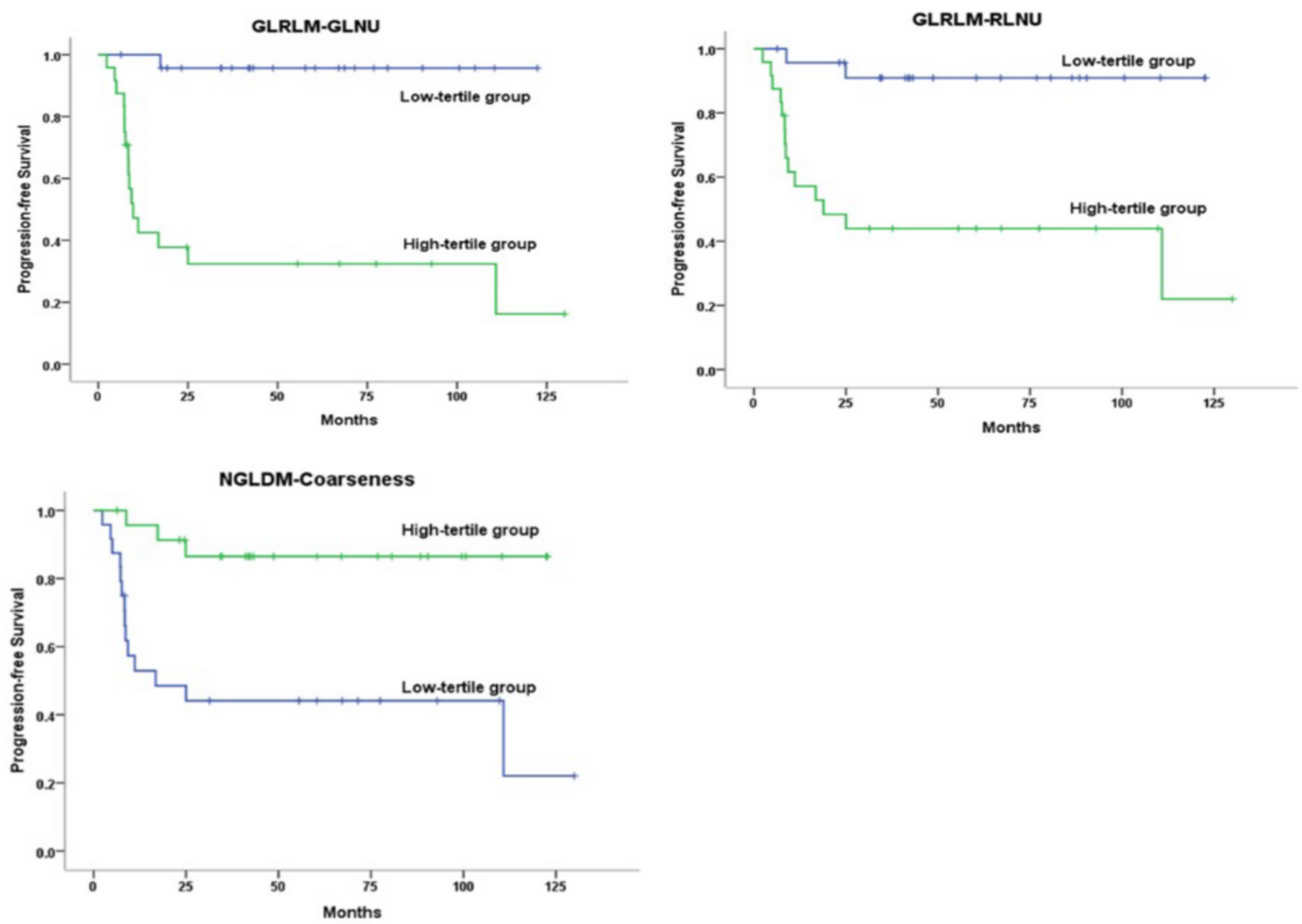

\section{Figure 4}

Progression-free survival of patients with low and high GLRLM-GLNU, GLRLM-RLNU and NGLDMcoarseness which are statistically significant textural features 